EPJ Web of Conferences 66, 10014 (2014)

DOI: $10.1051 /$ epjconf/ 20146610014

(C) Owned by the authors, published by EDP Sciences, 2014

\title{
Nondestructive measurement of environmental radioactive strontium
}

Shuntaro Saiba ${ }^{\text {a }}$, Tomohiro Okamiya, Saki Tanaka, Ryosuke Tanuma, Yumi Totsuka and Jiro Murata

Department of Physics, Rikkyo University, Tokyo 171-8501, Japan

\begin{abstract}
The Fukushima Daiichi nuclear power plant accident was triggered by the 2011 Great East Japan Earthquake. The main radioactivity concerns after the accident are I-131 (half-life: 8.0 days), Cs-134 (2.1 years), Cs-137 (30 years), Sr-89 (51 days), and Sr90 (29 years). We are aiming to establish a new nondestructive measurement and detection technique that will enable us to realize a quantitative evaluation of strontium radioactivity without chemical separation processing. This technique is needed to detect radiation contained in foods, environmental water, and soil, to prevent us from undesired internal exposure to radiation.
\end{abstract}

\section{Introduction}

Most of the radioactive materials, such as cesium isotopes, released from the nuclear power plant accident are gamma-emitting nuclei, whose radioactivity is relatively easy to determine by gammaspectroscopy using germanium detectors or conventional scintillation counters/survey meters. On the other hand, detection of Sr-90 by gamma-spectroscopy is very difficult because of its tiny gammadecay branching ratio of about $0.01 \%$. Therefore, only a small number of food and soil samples were measured after the accident, by using the standard chemical separation procedure which normally requires at least two weeks to obtain the Sr-Y radio-equilibrium. According to the governmental largescale surveying project led by MEXT around the power plant, the relative abundance of Cs- 134 to Cs137 was confirmed to be almost constant; however, that of Sr-90 to Cs-137 was found to widely vary from approximately $0.01 \%$ to $10 \%$ [1]. Therefore, we cannot make a reliable estimate of the strontium radioactivity by combining the measured value of cesium radioactivity with a presumed constant abundance ratio. Direct measurement of strontium radioactivity is hence required.

The half-life of Cs-137 is as long as that of Sr-90; however, their biological half-lives are very different (Cs-137: 3 months, Sr-90: 50 years) because of the differences in their chemical properties. Indeed, strontium isotopes are easily replaced with calcium and absorbed by bones, where they are only released with difficulty. Therefore, a quantitative determination of the environmental strontium radioactivity is important because of the substantial effect the radioactivity has on our lives.

\footnotetext{
${ }^{\text {a }}$ Corresponding author: saiba@rikkyo.ac.jp
} 


\section{Principle and Setup}

In our present study, Sr-90 radioactivity is measured by performing physical beta-spectroscopy utilizing the relatively large Q_beta of Y-90 (half-life: 64 hours; Q_beta: $2.3 \mathrm{MeV}$ ), which is the daughter nucleus of Sr-90. To perform the beta-spectroscopy using plastic scintillation counters, we need to be careful to account for the contribution to the plastic scintillation from the gamma-ray triggered events from Cs-134 and Cs-137 mixed in the samples, such as Compton scattering and photoelectron effect in the sample and detectors. The cesium-origin gamma-ray contributions are estimated and subtracted to extract the pure beta events by dedicated measurements surrounding the sample with an aluminum plate thick enough to stop all the beta-rays. The obtained beta spectra are then fitted with a summation of this gamma-contribution and a Y-90 beta spectrum, which is obtained by a Monte Carlo simulation including self-adsorption effect inside the sample.

The soil samples collected in Fukushima prefecture were contained in standard U-8 plastic containers $(100 \mathrm{ml})$ after mixing well and drying. In addition to beta measurements, we also performed gamma measurements using a germanium detector in a separate measurement for the same samples to estimate the cesium and strontium radioactivity. In this procedure, we need to be careful that only a surface region less than $1 \mathrm{~cm}$ contributes to the measured beta spectrum, whereas the entire sample volume contributes to the gamma spectrum.

As shown in Figure 1, a 1.0-cm-thick plastic scintillation counter is used to measure the beta energy, combined with a 2-mm-thick triggering (TRG) plastic scintillation counter, which suppresses gammaray events by requiring a coincidence with the $1 \mathrm{~cm}$ counter. VETO counters surrounding the setup are also used to suppress cosmic ray contributions. Although most of the direct gamma-ray events are rejected by requiring a coincidence with the trigger counter, a small fraction of them cannot be discriminated because of the Compton scattering events that behave like beta events.

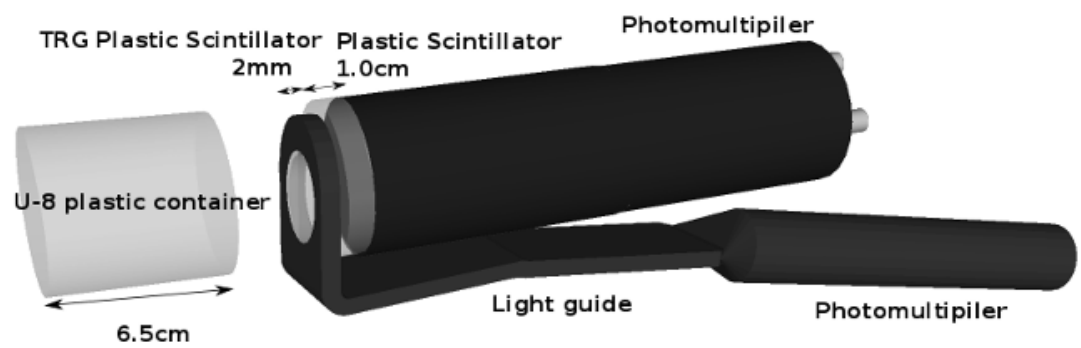

Figure 1. Detector setup

The Y-90 beta spectrum that is used to fit and to extract the strontium radioactivity is generated by a Monte Carlo simulation, which includes energy loss and Coulomb multiple-scattering processes. We have performed many test measurements to check the reliability of the Monte Carlo simulation code by comparing the results from measurements using thin plate-shaped soil absorbers with a superficial Sr-90 source. As shown in Figure 2, we confirmed that the prediction of the Monte Carlo simulation matches with the obtained test measurement results. Then, the expected beta spectrum from the volume source was generated using the Monte Carlo simulation in order to be used to fit the soil data. 


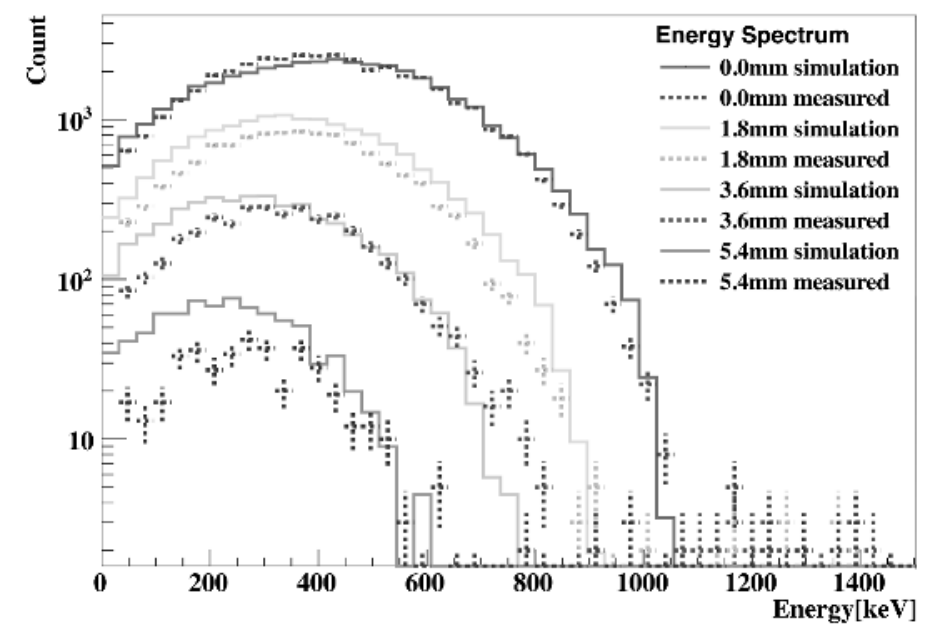

Figure 2. Comparison between the Monte Carlo and experimental data for thin soil-plates are shown.

\section{Results}

Beta spectra were measured with and without aluminum beta absorbers set between the sample and the detectors to estimate the gamma-origin contributions. Most of the spectrums with absorber were considered to be dominated by the photoelectric and the Compton scattering events. A small difference between them can be confirmed in Figure 3, indicating a successful detection of the real beta contribution. The results were fitted using the gamma-origin spectrum obtained from the "with absorber" spectrum and the Monte-Carlo-simulation-generated Y-90 spectrum. The calibration was performed by using a surface $\mathrm{Sr}-90$ beta source, whose activity was known to be $2.5 \mathrm{kBq}$. The geometrical effect from the effective volume contribution on the measured beta spectrum was corrected. We performed this measurement for two samples, 1 and 2. As a result, Sr-90 radioactivity was obtained as $0.9 \pm 0.2 \mathrm{kBq} / \mathrm{kg}$ for sample 1 and $0.5 \pm 0.3 \mathrm{kBq} / \mathrm{kg}$ for sample 2 .

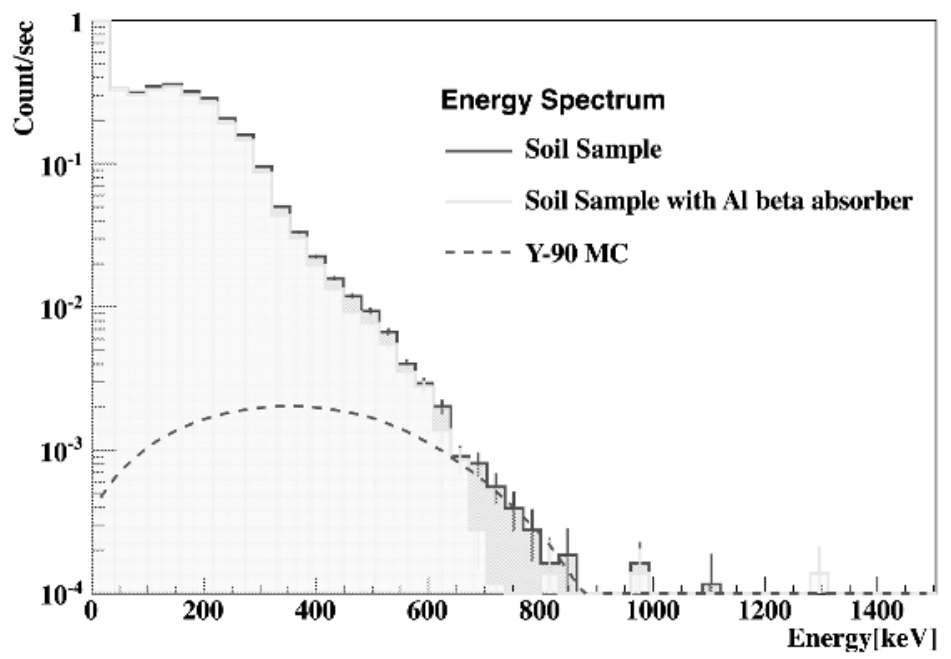

Figure 3. Obtained beta spectrum of the soil sample, for with and without aluminum beta absorber. The Monte Carlo spectrum of the Y-90 used for fitting is also shown as the dashed line. 
The same samples were analyzed using the standard radiochemical method performed by NKBC [2] to calibrate our results. In Figure 4, the results from our physical method and from NKBC's radiochemical method are compared, which shows a clear correlation between them. This result indicates that our method is sensitive to the strontium radioactivity, although disagreements on the absolute value need to be investigated.

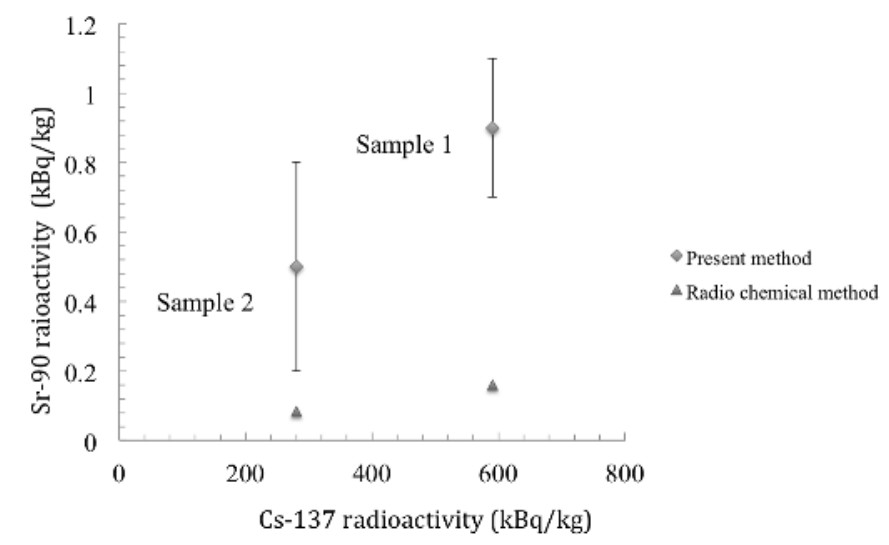

Figure 4. Comparison between present measurements and radio chemical method

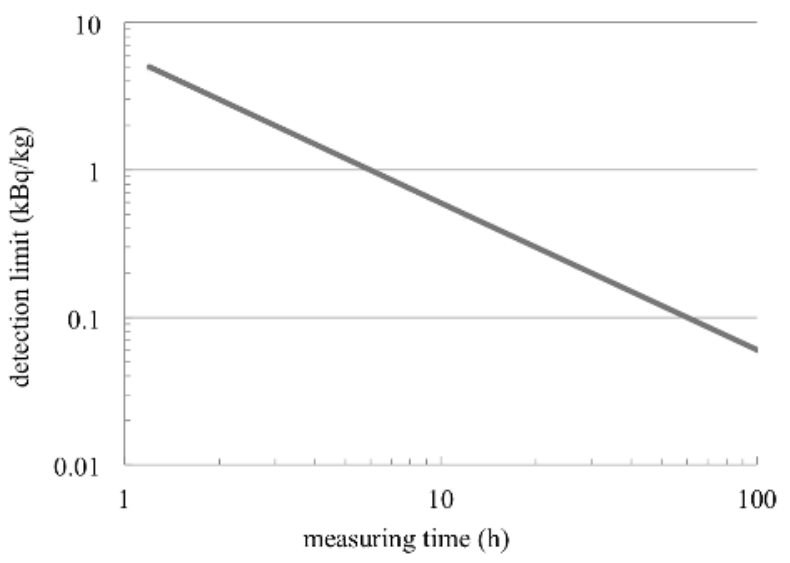

Figure 5. Detection sensitivity of the present method

A possible reason for the overestimation could be underestimation of gamma-ray events, which is considered the same as the with aluminum absorber measurement. The photoelectric and Compton scattering events inside the aluminum absorber, which we do not take into account in the present study, should be estimated in order to make an accurate subtraction. In the current setup, the detection limit obtained is $0.5 \mathrm{kBq} / \mathrm{kg}$ in a $12 \mathrm{~h}$ measurement period. This technique will be applied in an animal surveying project in Fukushima.

\section{References}

1. Monitoring information of environmental radioactivity level, Ministry of Education, Culture, Sports, Science and Technology, JAPAN;

http://radioactivity.mext.go.jp/ja/contents/7000/6213/24/338_0912_18_rev0914.pdf (2012)

2. Niigata-Prefecture Environmental Analysis Center: http://www.nkbc.jp/ 